\title{
A PLEA FOR AN EARLIER DIAGNOSIS OF CANCER OF THE UTÉRUS.
}

\author{
By F. WEBB GRIFFITH, M.D., \\ Late of the Gynecological Staf of Johns Hopkins Hospital. \\ Asheville, N. C.
}

From time inmemorial there has been no disease which has so persistently baffled the medical profession and which has been regarded as so hopeless by the laity as malignant growths.

Considering the enormous amount of time which has been spent in the past decade in the searcl for the cause and specific treatment of cancer, our knowledge is indeed meager. :

Because of our inability to cure moderately advanced cases of carcinoma and because of the morbid depression produced upon the patient, they have become a harvest-field for the quack.

In gynecological work cancer of the uterus so overshadows all other forms of malignancy that I have chosen it as the subject of this paper.

In Europe the medical profession, under the guidance of Winter and others, have, through the medium of the press and circulars, so impressed upon the women the importance of an early recognition of cancer of the womb that already the results are marvelous.

In this country, however, public opinion would be against any such method, and we must depend entirely upon the family plysician.

The uterus is composed of two portions, the corpus or bolly and cervix or neck, and in the female economy these two portions have absolutely different functions to perform. The inner lining of both the corpus and the cervix is composed of a single layer of cylindrical epithelial cells. The cells in the corpus differ slightly from those in the cervix, but for practical purposes this single layer of cells extends from the top of the corpus to the external os of the cervix.

Now, extending from the external os of the cervix around the outer side of the cervix, and covering the vagina, are several layers of epithelium. Just as there are two kinds of epithelium in and around the uterus, so there are two kinds of carcinoma or cancer depending upon the origin. The one arising from the single layer of epithelium, and known as adeno-carcinoma, the other arising from the outer many-layered epithelium and known as squamous-celled carcinoma.

Thus we have adeno-carcinoma arising either from the inner lining of the cervix or from the inner lining of the body, whereas squanous-celled carcinoma practically always arises from the cervix, for there are no squamous or many-layered cells in the body of the uterus.

As to rapidity of growth, those arising from the inner layer of the cervix (the adeno-carcinomata of the cervix) are by far the most actively growing. The next in rapidity of growth are those arising from the outer wall of the cervix, the squamous-celled carcinomata. While the least rapidly growing are those arising from the inner lining of the body of the uterus, the acieno-carcinomata of the body.

These three types will be discussed more in detail later when we consider the symptoms.

The cause of cancer is unknown. Many attractive theories have been advanced to explain it, but as yet none have been proven.

The frequency with which a history of injury or chronic irritation is obtained in carcinomata of the breast, lips and other portions of the body would seem to indicate that trauma is an important etiological factor. Certainly it is that cancer of the cervix in a woman who has had neither children, miscarriages or instrumental manipulations of the uterus, is un-

\footnotetext{
* Read before the County Medical Society. Asheville, N. C., November 20, $19 t 1$.
} 
common. One authority states that only 3 per cent of the cases of carcinomata of cervix had never been pregnant, while that 42 per cent of the cases of carcinomata of the body of the uterus were in nullipara. So that. whatever may be said of tramma as an etiological factor of cancer elsewhere in the body, experience show's that it is of great importance in cancer of the cervix, while in the body oi the uterus it seens to have no significance.

The belief that heredity is the cause of cancer is deeply rooted in the minds of the laity. In the past few years several women without any symptoms have come to be to be examined, fearing that thes miglit have cancer of the womb because their mother or sister had died of it.

There are on record many fanilies in which several members liave died of the same kind of malignant trouble at or about the same age. This does not by any means prove hereditary influence, for when we consider how common are malignant growths, it would be strange if such occasional coincidences did not occur.

Parasites, embryonic misplacements. "break in the continunity of cells," are but theories to account for the origin of cancer and beyond the scope of this paper.

Cancer of the uterine cervix is a disease of middle adult life. It is extremely rare in youth and very infrequent in old age. Between the ages of thirty and fity years it is common, being most apt to occur at or about the menopause.

Cancer of the hody of the uterus tends to occur a little later in life, being most common between forty and sixty years of age.

Since cancer of the cervix, when moderately advanced, offers such little hope of cure, it behooves the specialist to learn to recognize it, and the general practitioner to at least suspect it in its incipiency.

- The two great symptoms of cancer of the cervix and danger signals to the woman near the menopause, are an unusual bleeding and discharge. Bleeding from any other portion of the body thain the uterus is always to a certain degree alarming, both to the patient and to the physician, yet uterine bleeding appearing each month as a menstrual flow seems to lose its power to impress us.

Any abnormal bleeding whatsoever in a woman over thirty-five years of age demands investigation. The bleeding of an early cancer may be intermenstrual, that is, occurring between the menstrual periods; it may be a profuse hemorrluge or it may be only enough to soil the clothing. On the other hand, the abnormal bleeding may be in the form of an increased menstruation. A woman who has previously menstruated regularly for three to four days will rather suddenly begin to menstruate for six to seven days, or she may menstruate her usual number of days, but in that time lose more blood than formerly.

As a rule, a woman with what seems to her such slight menstrual disturbances, will not consult a physician, and if she does the physician will often pass it by lightly, assuring her that everything will soon be all right. IVe all recognize the prejudice a woman has against examination, and the difficulties and inconveniences of making pelvic examinations, but when we consider the hundreds of women who are pouring annually into the hospitals only to receive the dreaded verdict, "hopelessly inoperable," it makes us realize that it is better to examine a score of women unnecessarily than to let one single operable case escape.

Another sad delusion which exists so frequently in the minds of women, is that the appearance of bleeding after the menopause is but the re-establishment of menstruation. Also that increased biceding between the ages of forty and forty-five is one of tine phenomena of the "cliange of life."

The menopause may be heralded by a decrease in the nenstrual flow, but never by an increase. Any increased or unusual bleeding around the menopause indicates that something is wrong.

I remember so vividly a woman, forty-seven 
years old, who told me that she believed she was getting young again, because her periods which had ceased a few years previously, were returning. Poor woman, she did not realize that she was the victim of an extensive cancer and had only a few months to live.

Let me give you briefly the history of one of our cases picked at random from the records of the Johns Hopkins Hospital, which shows the hopeless condition in which most of the cases of cancer of the cervix are received at this institution. Mrs. B. L., aged $3^{8}$. white, admitted with the following history: Menstruation began at 13 years of age, always regular, lasting five to six days, with practically no dysmenorrhea. For the last four or five years the flow has been increasing in amount but not in duration. (Notice that for over four years the flow had been increasing.) Patient has been married twentythree years and had had five children and two miscarriages. Always a strong woman, with never a day's sickness except at chilk-birth. Her illness began sixteen months before admission, when, while doing some heary lifting. she became unwell. Her flow lasted five weeks, but she paid no especial attention to it. It finally changed to a sero-sanguinous discharge, which persisted to time of admission, with, however, three severe hemorrhages in the meantime. Soon after the onset her doctor told her she had a tumor and that her womb should be scraped, which advice she followed. Her doctor, whoever he was, certainly deserves credit for urging an early curettage. but unfortunately a fatal error was made, for the scrapings were not cxamined microscopically, or else were examined by someone untrained in gynecological pathology, for the malignant nature was overlooked.

After the curettage the patient became markedly worse, and on admission to the hospital was suffering a great deal of pain, was weak and had lost about sixty-five pounds in weight.

The following note was dictated on her admission to the hospital, sixteen months after the appearance of marked and conspicuous symptoms and over four years after the beginning of an increased menstrual flow: "External genitalia normally developed. Outlet parous and relaxed. Very foul-smelling bloody seropurelent discharge. Inspection showed vault of vagina occupied by fungating mass. Palpation reveals cervix entirely replaced by spongy, friable, fungating mass extending up into body of uterus and over vaginal vault above and to left to within three centimeters of outlet, on right not quite so far. Mass infiltrates surrounding tissues deeply, is quite immovable and fixed on left and slight movement possible on right. Rectal examination gives intimate connection between mass and rectum.

Diagnosis: "Carcinoma cervicis uteri. Condition clearly inoperable."

Surprising as it may seem to you, that is about the condition in which are over half the patients with cancer of the cervix who apply for admission to this hospital.

Contrast the above history with one of our very early cases. Mrs. S. C., aged 48 , white, admitted June, 1908. Menstruation regular every four weeks until September, 1907, when she stopped for five months, that is, until February, Igo8, when she menstruated her usual length of time. Then she did not menstruate again until May, when she had two periods which were very profuse. There you see in May, I908, was the first actual increase of bleeding. She consulted her physician, who, although he could find nothing wrong on examination, realized fully the danger of allowing any increased bleeding in a woman 48 years old to go uninvestigated, sent her into the hospital immediately for the advice of a specialist.

Under an anaesthetic the following condition was found: Cervix quite soft. There was a small amount of bleeding coming from the cervix, but there was no evidence of any induration or ulceration or loss of substance in the cervix to suggest a malignant growth. 
The uterus was in anteposition, about normal size and symmetrical. No tumors could be palpated at any point. The lateral structures were normal.

The uterus was curetted for diagnosis, and the uterine scrapings found to be definitely carcinomatous, but very early. The uterus was then removed and the growth found to be localized. The patient has since remained well and we have every reason to believe she will continute so.

This case well illustrates what I wish to emphasize, that although the physician or specialist may see or feel absolutely nothing abnormal, yet any increased bleeding or discharge near the menopause demands the most thorough investigation. This patient undoubtedly owes her life to her family physician, and this one case alone would abundantly compensate hin for any number of other suspicious cases whom he may have submitted to examination or even curettage. unnecessarily.

Cancer of the womb, although most commonly of cervical origin, does in about 20 per cent of the cases arise from the fundus or body: Unfortumately this relative percentage is so small, for cancer of the fundus offers very much better immediate and ultimate prognosis thain cancer of the cervix. For that reason operation several months or even a year after the onset of symptoms may offer a reasonable hope of cure, while a cervical carcinoma of that duration would be practically hopeless. Because of the slow growth of cancer of the body, of the uterus, the family physician should be the more enger to get it early, within the first few weeks, for then it offers an excellent clance for an absolute cure.

Just as in cancer of the cervix, so in cancer of the fundus, unusual bleeding and unusual discharge are the two important early symptoms which should be indelibly impressed on the nind of the physician. And as far as the general practitioner is concerned, these two symptoms are all he need bother about so long as he attaches to them the importance they deserve. For when the other manifestations, such as severe pain of bladder or rectal involvement occurs, it is too late.

There are certain benign conditions which may simulate cancer of the womb and mislead the physician. If he will consider every suspicious cause malignant until proven otherwise, he will frequently err, 'tis true, but his error will always be on the safe side and not at the expense of a human life.

What are some of these conditions which may deceive us? First we will consider the so-called ulceration of the neck of the womb.

Only recently Dr. Howard Kelly stated that in his whole experience he had seldom seen a true ulceration of the cervix, that is, where there was actual loss of substance, which was not malignant, except in prolapse where the cervix was irritated by clothing, thighs or some external agent. In those cases of complete prolapse, clothing, irritation by rubbing against the thighs, etc., cause slight abrasion in the cervical mucosa, which become infected, with a resultant loss of tissue.

Although these cases are usually fairly easy to differentiate from cancer, yet the constant irritation and trauma seem to be exciting or predisposing factors to the formation of a malignant growth, and for that reason they should receive proper treatment.

If an authority like Kelly states that a true benign ulceration of the cervix is so extremely rare, when, then, is the condition which the general practitioner so frequently diagnoses as ticeration of the neck of the womb?

You recall that the lining of the cervical canal is composed of a single layer of epithelial cells, while the outer or vaginal portion of the cervix is covered by several layers. Now, beneath the epithelium are numerous bloodvessels, which, slinnig through the single layer of cells, gives to it a "beefy-red" appearance. On the saginal portion of the cervis, that is, outside of the external os, where there are many layers of cells, the vessels do not 
shine through so distinctly, but give rather a pinkish-blue appearance.

At the time of labor it is quite common to get a bilateral laceration of the cervix and consequent eversion or turning out of the anterior and posterior lips. So that when the physician looks at the cervix he sees around the external os a rim about $1 / 4$ to $1 / 2$ inch in diameter of beefy, angry appearance. which is, in reality, nothing but the normal cervical lining exposed to view owing to the cervical laceration, and is not an ulceration or loss of tisstie.

Once in a while we find a somewhat similar condition in a nullipara, where there has been no laceration or eversion of the cervix, but where the junction between the single layer of cervical epithelium and the many-layered vaginal epithelium is not as normally at the external os, but farther out on the vaginal portion of the cervix. This is a congenital anomaly and relatively infrequent.

If a physician applies some caustic to this "supposed tulceration" there will be a proliferation of cells due to the irritation, and consequently the vessels not showing through so distinctly, this area takes on the appearance of the rest of the vagina and the "ulceration" is said to be healed. After a few weeks or months these proliferated cells will desquamate and the ulceration is said to have recurred, and the caustic is again applied. Often women with vague pelvic complaints will be subjected to repeated cauterization of the cervix on account of this so-called "ulceration," when, as a matter of fact, it seldom causes symptoms and should not have caustic applications.

I have digressed a little by considering in detail eversion of the cervix, but it is because this subject of so-called cervical ulceration is universally misunderstood by the general practitioner, and because in many cases, it is of great vaue in differential diagnosis.

When a woman near the menopause presents what seems to be an ordinary cervical erosion, but has also an unusual discharge or bleeding, the condition should by all means be considered malignant until proven otherwise.

The second benign condition which sinulates malignancy is myoma, for one of the symptoms of a uterine myoma which involves the endometrium, is bleeding. If now a woman near the menopause has such a myomatous nodule, yet too small to be felt by bimanual examination, she will have bleeding of obscure origin.

A polypus projecting into the uterine cavity will likewise give bleeding, the cause of which cannot be detected by the ordinary examination.

A threatened abortion before the second month in a woman near the menopause is sometimes very difficult to distinguish from an early malignancy. If the presumptive signs of pregnancy, such as changes in the breasts, blueing of the vagina, softening of the cervix, etc., are present, of course the diagnosis is simple. But if. as so otten happens, these signs are vague or even absent, and especially if leucorrhea, which is so common in a pregnant woman, is present, you have three of the characteristics of a malignant growth of the body of the uterus, bleeding, leucorrhea and an enlarged uterus.

The fifth benign condition simulating cancer is the retention of a portion of the membranes after an abortion, when the patient is near the menopause.

As you know, if after an abortion there remains in the uterine cavity a small piece of the membranes, a persistent bleeding will be kept up until the offending tissue either disintergrates and is cast off or else is curetted away.

When there is a clear history of early pregnancy and abortion, the diagnosis of retained membranes offers no difficulty.

The cases which are confusing will come with a history somewhat like this: A woman between the ages of 40 and 45 misses a menstrual period, and she does not know whether it is due to an early pregnancy or to "change 
of life." Mhile doings some heavy lifting or unusual exertion, she will have a profuse bleeding lasting from a few hours to a day or so and followed by a blood-tinged discharge for several weeks, when she will finally decide to consult a physician. The question then arises. has she had an incomplete ahortion at about the fourth or fifth week, or has she an early cancer?

Without a curettage of the uterus it is impossiblc to make an absolute diagnosis. Fortumately. however. if it be retained membranes, curettage is the treatment indicated. whereas if it he cancer the same procedure is necessary for dingnosis.

There are other raver benign conditions which might be nistaken for an early cancer of the womb. but I will not tire you by a recital of rareties which the general practitioner selklom encounters.

Thus we have, on the one hand, a large group of pathological processes, some of which would, in the course of time, tend to correct themselves, but we have, on the other hand, with practically the same symptoms, cancer of the womb, where delay means death.

If any of us shouli treat a case of suspected diphtheria for a week without taking cultures from the throat to determine its nature, or administering antitoxin, and the patient should die. we could be held responsible for criminal neglect. Yet we allow a woman with bleceling and discharge suspicious of cancer, to go week after week and month after month until anaemic from the loss of blood and cachectic from the toxins of cancer, she is beyond hope.

What, then, is the course why the pliysician should follow in these suspected cases?

If he has lad training in aseptic technique, he may dilate the cervix and curette the uterine cavity, and send the curettings preserved in alcohol or to per cent formalin, to a pathologist for diagnosis.

If there be a malignant growtil, it is worth infinitely many times the trouble and cost to catch it early, and if it should not be malignant, it will be a great comfort to be able to reassure the patient.

If the patient is poor and cannot afford to pay a specialist, there is no gynecologist worthy of the name who would not gladly treat her gratis.

So that it simply resolves itself into whether the physician and patient are willing to put themselves to the slight trouble and inconvenience of seeing a specialist, and surely that could be no argunent for delay when a human life is at stake.

I firmly believe that this generation will witness the discovery of the cause of cancer and possibly also its cure in moderately advanced cases. Whether the treatment then will be with drugs, serum or scalpel, we can only surmise. but with our present knowlerlge mighty little can be done toward a cure unless gotten at its onset.

78 Patton sa'cunc.

\section{A VITALIA IMPORTANT DISTINCTION.}

In the Therapentic Guzelle of Jamury 15, 1912, is a thoughtful article suggested by expressions in the Neai Fork Medical Journal. The following brief abstract is pregnant with important facts demanding the thoughtul consideration of every conscientious physician:

Is is well known, there are three important vascuhar areas which are not controlled, or only very weakly controlled, by vasoconstrictor nerves, the brain, lungs, and heart. Adrenalin has little, if any, constricting action upon the vesșels of these organs: it is said to be almost useless in operations on the brain. The ressels of these areas are, however, contrac:ed by pituitary extract. After a very brisf period of stimu'ation the heart is weakened by gituitary extract: this effect seems to be due to the constriction of the coronary vessels. The weakening of the heart from pituitary extract causes a fall of pressure in the pulmonary circuit; the general vaso-constriction causes a rise in systemic pressure which counteracts the tendency to anemia of the medulary centers which results from cardiac depressants. Wiggers (Archices of Internal Medicinc. July 15, 1911), believes that this combination of actions peculiarly adapts the pituitary extract for !se in hemoplysis. The fact that adrenalin, through its stimulating action on the heart, increases the pressure in the pulmonary circuit would contraindicate its l1se in certain cases of pulmonary hemorrhage. 